\title{
Image Classification Using Separable Discrete Moments of Charlier-Tchebichef
}

\author{
Mhamed Sayyouri, Abdeslam Hmimid, and Hassan Qjidaa \\ CED-ST; LESSI; Faculty of sciences Dhar El Mehraz, \\ University Sidi Mohamed Ben Abdellah BP 1796 Fez-Atlas 30003, Fez, Morocco \\ \{Mhamedsay, abdeslam_ph, qjidah\}@yahoo.fr
}

\begin{abstract}
In this paper, we propose a new set of Charlier-Tchebichef invariant moments. This set is derived algebraically from the geometric invariant moments. The presented approach is tested in several well known computer vision datasets including moment's invariability and classification of objects. The performance of these invariant moments used as pattern features for a pattern classification is compared with Tchebichef-Krawtchouk, Tchebichef-Hahn and Krawtchouk-Hahn invariant moments.
\end{abstract}

Keywords: Harlier-Tchebichef invariant moments, classification, pattern recognition.

\section{Introduction}

The theory of moments has been widely used for image analysis and pattern recognition [1-5]. Hu [1] has derived a complete system of geometrical moment invariants under the transformations of translation, scaling, and rotation of the image. This set of moments is not orthogonal which causes the redundancy of information. To overcome this problem, the continuous orthogonal moments as Legendre [2], Zernike [2], Gegenbauer [3] and Fourier-Mellin [4] are introduced in the fields of image. The orthogonal property of continuous orthogonal moments assures the robustness against noise and eliminates the redundancy of information [2-4], but their computation requires the discretization of continuous space and the approximation of the integrals which increases the computational complexity and causes the discretization error [5-9]. To eliminate this error, the discrete orthogonal moments such as Tchebichef [8], Krawtchouk [9], Charlier [10] and Hahn [11-13] have been introduced in image analysis and pattern recognition. The use of this set of moments satisfies exactly the orthogonal property and eliminates the need for numerical approximation [14-15].

Recently, a novel set of discrete and continuous orthogonal moments based on the bivariate orthogonal polynomials have been introduced into the field of image analysis and pattern recognition [16-18]. In this paper, we present a new set of discrete orthogonal moments based on the product of Charlier and Tchebichef discrete orthogonal polynomials which are denoted Charlier-Tchebichef moments (CTM). The paper also proposes a new set of discrete invariant moments of Charlier-Tchebichef 
(CTMI) under translation, scaling and rotation of the image. The CTMI is derived algebraically from the geometric invariant moments. The accuracy of object classification by our descriptors CTMI is compared with Tchebichef-Krawtchouk invariant moments (TKMI) [16], Tchebichef-Hahn invariant moments (THMI) [16] and Krawtchouk-Hahn (KHM)[16] invariant moments.

The rest of the paper is organized as follows: In Section 2, we present the known results of the Charlier and Tchebichef discrete orthogonal polynomials with one variable. Section 3 presents the definition of CTM moments. Section 4 focuses on the deriving of CTMI from the geometric moments. Section 5 provides some experimental results concerning the invariability and objects classification by CTMI. Section 6 concludes the work.

\section{Classical Discrete Orthogonal Polynomials}

In this section, we will present a brief introduction to the theoretical background of classical discrete orthogonal polynomials with one variable of Tchebichef and Charlier [19-20].

\subsection{Tchebichef's Polynomials}

The $\mathrm{n}^{\text {th }}$ Tchebichef polynomial is defined by using hypergeometric function as:

$$
t_{n}(x ; N-1)=(1-N)_{n 3} F_{2}(-n,-x, 1+n ; 1,1-N ; 1)=\sum_{i=0}^{n} \delta_{m, i} x^{i}
$$

The normalized discrete orthogonal polynomials of Tchebichef are defined by:

$$
\tilde{t}_{n}(x ; N-1)=t_{n}(x ; N-1) \sqrt{\frac{1}{\rho_{t}(n)}}
$$

with $\rho_{t}(n)$ is the squared norm of Tchebichef polynomials defined as:

$$
\rho_{t}(n)=\frac{(N+n) !}{(2 n+1)(N-n-1) !}
$$

\subsection{Charlier's polynomials}

The $\mathrm{n}^{\text {th }}$ discrete orthogonal polynomial of Charlier $C_{n}^{a_{1}}(x)$ is defined by using hypergeometric function as [20]:

$$
C_{n}^{a_{1}}(x)={ }_{2} F_{0}\left(-n,-x ;-; 1 / a_{1}\right)=\sum_{k=0}^{n} \alpha_{k, n}^{\left(a_{1}\right)} x^{k} \quad ; x=0,1,2 \ldots \quad ; n \geq 0 \quad ; \quad a_{1}>0
$$

The normalized discrete orthogonal polynomials of Charlier are defined by: 


$$
\tilde{C}_{n}^{a_{1}}(x)=C_{n}^{a_{1}}(x) \sqrt{\frac{w_{c}(x)}{\rho_{c}(n)}}
$$

where $\rho_{c}(n)=\frac{n !}{a_{1}^{n}}$ and $w_{c}(x)=\frac{e^{-a_{1}} a_{1}^{x}}{x !}$

\section{Charlier-Tchebichef Moments}

The two-dimensional (2-D) Charlier-Tchebichef's discrete orthogonal moments (CTM) of order $(\mathrm{n}+\mathrm{m})^{\text {th }}$ of an image intensity function $f(x, y)$ with size $M \times N$ is defined as:

$$
C T M_{n m}=\left[\rho_{c}(n) \rho_{t}(m)\right]^{-1 / 2} \sum_{x=0}^{M-1} \sum_{y=O}^{N-1} \tilde{C}_{n}^{a_{1}}(x) \tilde{t}_{n}(y, N-1) f(x, y)
$$

with $\tilde{C}_{n}^{a_{1}}(x)$ and $\tilde{t}_{n}(y, N-1)$ are the nth order of orthonormal polynomials of Charlier and Tchebichef respectively.

\section{Charlier-Tchebichef's Invariant Moments}

\subsection{Geometric Invariant Moments}

Given a digital image $f(x, y)$ with size $\mathrm{M} \times \mathrm{N}$, the geometric moments $G M_{n m}$ are defined using discrete sum approximation as:

$$
G M_{n m}=\sum_{x=0}^{M-1} \sum_{y=0}^{N-1} x^{n} y^{m} f(x, y)
$$

The set of geometric invariant moments (GMI) by rotation, scaling and translation can be written as [1]:

$$
G M I_{n m}=G M_{00}^{-\gamma} \sum_{x=0}^{M-1} \sum_{y=0}^{N-1}[(x-\bar{x}) \cos \theta+(y-\bar{y}) \sin \theta]^{n}[(y-\bar{y}) \cos \theta-(x-\bar{x}) \sin \theta]^{m}
$$

with

$$
\bar{x}=\frac{M G_{10}}{M G_{00}} ; \bar{y}=\frac{M G_{01}}{M G_{00}} ; \gamma=\frac{n+m}{2}+1 ; \theta=\frac{1}{2} \tan ^{-1} \frac{2 \mu_{11}}{\mu_{20}-\mu_{02}}
$$

The $(n+m)^{\text {th }}$ central geometric moments is defined in [1] by:

$$
\mu_{n m}=\sum_{x=0}^{N-1} \sum_{y=0}^{M-1}(x-\bar{x})^{n}(y-\bar{y})^{m} f(x, y)
$$




\subsection{Computation of Charlier-Tchebichef's Invariant Moments}

To use the Charlier-Tchebichef's moments for the objects classification, it is indispensable that be invariant under rotation, scaling, and translation of the image. Therefore to obtain the translation, scale and rotation invariants moments of CharlierTchebichef (CTMI), we adopt the same strategy used by Author et al. for Hahn's moments in [12]. That is, we derive the CTMI through the geometric moments.

The Charlier-Tchebichef moments of $f(x, y)$ can be written in terms of geometric moments as:

$$
\begin{aligned}
C T M_{n m} & =\left[\rho_{c}(n) \rho_{t}(m)\right]^{-1 / 2} \sum_{x=0}^{M-1} \sum_{y=0}^{N-1} c_{n}^{a}(x) t_{m}(y, N-1) f(x, y) \\
& =\left[\rho_{c}(n) \rho_{h}(m)\right]^{-1 / 2} \sum_{i=0}^{n} \sum_{j=0}^{m} \alpha_{n, i}^{\left(a_{1}\right)} \delta_{m, j} G M_{i j}
\end{aligned}
$$

The Charlier-Tchebichef's invariant moments (CTMI) can be expanded in terms of GMI as follows:

$$
\operatorname{CTMI}_{n m}=\sum_{i=0}^{n} \sum_{j=0}^{m} \alpha_{n, i}^{\left(a_{1}\right)} \delta_{m, j} V_{i, j}
$$

where $\delta_{m, j}$ and $\alpha_{n, i}^{\left(a_{1}\right)}$ are the coefficients relative to Eq.(1) and Eq. (4) and $V_{i, j}$ are the parameters defined as:

$$
V_{n m}=\sum_{q=0}^{n} \sum_{p=0}^{m}\left(\begin{array}{l}
n \\
p
\end{array}\right)\left(\begin{array}{l}
m \\
q
\end{array}\right)\left(\frac{N \times M}{2}\right)^{((p+q) / 2)+1} \times\left(\frac{N}{2}\right)^{n-p} \times\left(\frac{M}{2}\right)^{m-p} \times G M I_{p q}
$$

\section{$5 \quad$ Results and Simulations}

In this section, we give experimental results to validate the theoretical results developed in the previous sections. This section is divided into two sub-sections. In the first subsection, we showed the invariability of CTM under the three transformations translation, scaling and rotation. In the second sub-section, the recognition accuracy of CTMI is tested and compared to other descriptions given in [16] for objects classification.

\subsection{Invariance}

In this section we test the invariance of Charlier-Tchebichef invariant moments under translation, scale and rotation of the image. For this we will use a gray-scale image "Cat" (Fig.1) whose size is 128x128 pixels chosen from the well-known Columbia database [21]. This image is scaled by a factor varying from 0.5 to 1.5 with interval 0.05 , rotated from $0^{\circ}$ to $360^{\circ}$ with interval 10 and translated by a vectors varying from $(-5,-5)$ to $(5,5)$. Each translation vector consists of two elements which represent a vertical and a horizontal image shift respectively. All invariant moments of CTMI is 
calculated up to order two for each transformation. Finally, in order to measure the ability of the CTMI to remain unchanged under different image transformations, we define the relative error between the two sets of invariant moments corresponding to the original image $f(x, y)$ and the transformed image $g(x, y)$ as:

$$
E_{C M}(f, g)=\frac{\|C T M I(f)-C T M I(g)\|}{\|C T M I(f)\|}
$$

where $\|$ denotes the Euclidean norm and $\operatorname{CTMI}(f)$; CTMI $(g)$ are invariant moments of Charlier-Tchebichef for the original image $f$ and the transformed image $g$.

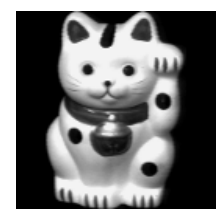

Fig. 1. Cat gray-scale image

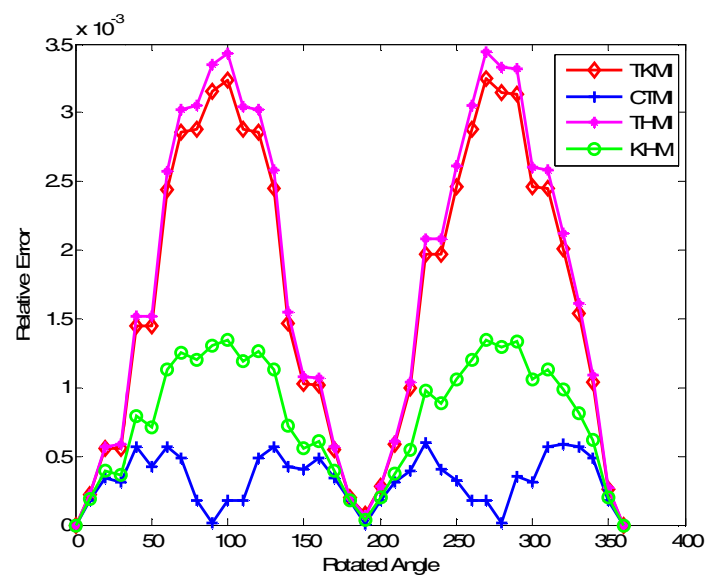

Fig. 2. Comparative study of relative error between the rotated image and the original image by CTMI, TKMI, THMI and KHMI

Figure 2 compares the relative error between the proposed invariant moments of CTMI, the invariant moments of Tchebichef-Krawtchouk TKMI [16], the invariant of Tchebichef-Hahn THMI [16], and the invariant of Hahn Krawtchouk-HKMI [16], relative to rotation of the image. It can be seen from this figure that the CTMI is more stable under rotation (very low relative error) and is better performance than the TKMI, THMI and KHMI, whatever the rotational angle.

Figure 3 shows the relative error between the CTMI, TKMI, THMI and KHMI relative to scale. The figure shows that, in most cases, the relative error of CTMI is more stable and lower than the TKMI, THMI and KHMI. 


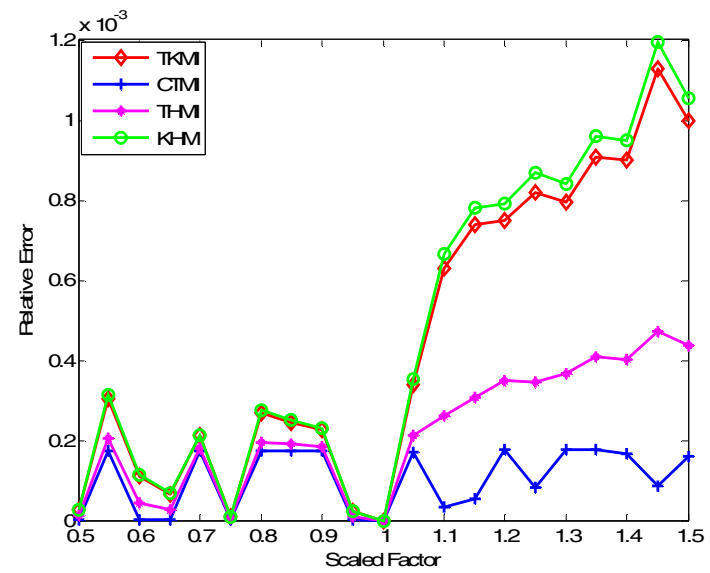

Fig. 3. Comparative study of relative error between the scaled image and the original image by CTMI, TKMI, THMI and KHMI

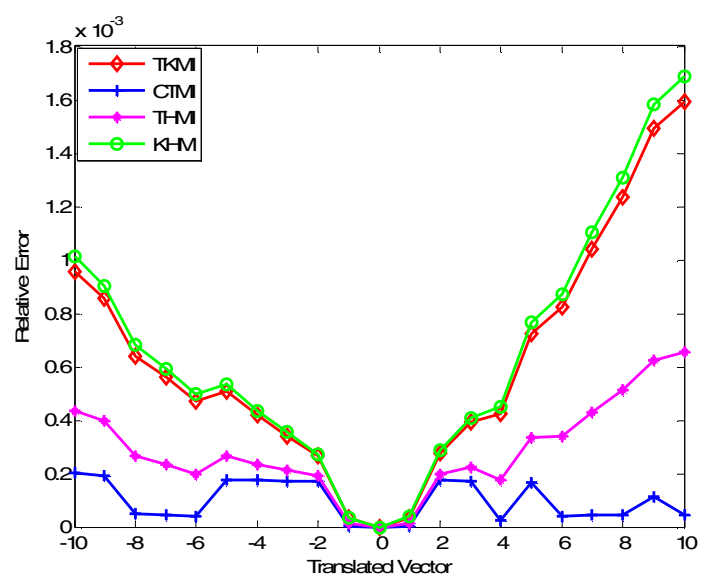

Fig. 4. Comparative study of relative error between the translated image and the original image by CTMI, TKMI, THMI and KHMI

Figure 4 shows the relative error between the CTMI, TKMI, THMI and KHMI relative to translation. The figure shows again that, in most cases, the relative error of CTMI is more stable and better performance than the TKMI, THMI and KHMI, whatever the translation vectors. Note that, the results are plotted in Figures (2, 3 and 4) for the case $a_{1}=80$ for the Charlier's polynomials, $p=0.5$ for Krawtchouk's polynomials and $a=b=10$ for Hahn's polynomials.

The results show that the CTMI is more s table under translation, scale and rotation of the image than the TKMI, THMI and KHMI. 


\subsection{Classification}

In this section, we will provide experiments to validate the precision of recognition and the classification of objects using the CTMI. For this, we will put in place the characteristic vectors defined by:

$$
V=\left[C T M_{i j}\right] ; i, j=0,1,2
$$

To perform the classification of the objects to their appropriate classes we will use simple classifiers based on Euclidean distances [23].

$$
d\left(x_{s}, x_{t}^{(k)}\right)=\sqrt{\sum_{j=1}^{n}\left(x_{s j}-x_{t j}^{(k)}\right)^{2}}
$$

The above formula measure the distance between two vectors where $x_{s}$ is the $\mathrm{n}$ dimensional feature vector of unknown sample, and $x_{t}^{(k)}$ the training vector of class $k$. If the two vectors $x$ and $y$ are equals, then $d(x, y)$ tend to 0 . Therefore to classify the images, one takes the minimum values of the distance.

We define the recognition accuracy as:

$$
\eta=\frac{\text { Number of correctly classified images }}{\text { The total of images used in the test }} \times 100 \%
$$

In order, to validate the precision of recognition and the classification of objects using the CTMI, we well use two image databases. These bases are standard bases used by the scientific community during the testing and validation of their approach and are freely available on the Internet. Each image database has defined the classes where each image belongs to one class. The first database is MPEG-7 CE-shape-1 Part [22]. This database contains 20 different binary images for 72 objects. Each image is resized in $128 \times 128$. This base has the characteristic of being widely used in image classification. The second image database is the Columbia Object Image Library (COIL-20) database [21]. The total number of images is 1440 distributed as 72 images for each object. All images of this database have the size 128x128. We tested the ability of classification of our descriptor CTMI compared to other descriptors of TKMI, THMI and KHMI [16] for the two databases. The test is followed by adding different densities of salt-and-pepper noise.

The results of Table 1 and Table 2 show the efficiency of the CTMI in terms of recognition accuracy of noisy images, compared to those of THMI, TKMI and KHMI. The comparison results shows the superiority of the proposed moments based on polynomials the Charlier and Tchebichef relative to moments based on the other polynomials. Note that the recognition of non-noisy binary image by our method is $100 \%$, and the accuracy of the recognition decreases with increasing noise.

Finally, the proposed CTMI are robust to image transformations under noisy conditions and the recognition accuracy. 
Table 1. Classification results of MPEG-7 CE-shape-1 database using Euclidean distance

\begin{tabular}{llllll}
\hline \multicolumn{5}{c}{ Salt \&pepper noise } \\
\hline & Noise free & $1 \%$ & $2 \%$ & $3 \%$ & $4 \%$ \\
\hline TKMI & $100 \%$ & $97.18 \%$ & $95.67 \%$ & $91.29 \%$ & $91.01 \%$ \\
THMI & $100 \%$ & $76.89 \%$ & $94.36 \%$ & $91.85 \%$ & $89.57 \%$ \\
KHMI & $100 \%$ & $97.19 \%$ & $95.81 \%$ & $92.03 \%$ & $90.14 \%$ \\
CTMI & $100 \%$ & $97.58 \%$ & $96.15 \%$ & $93.64 \%$ & $91.47 \%$ \\
\hline
\end{tabular}

Table 2. Classification results of COILL-20 objects database using Euclidean distance

\begin{tabular}{llllll}
\hline \multicolumn{5}{c}{ Salt \&pepper noise } \\
\hline & Noise free & $1 \%$ & $2 \%$ & $3 \%$ & $4 \%$ \\
\hline TKMI & $96.57 \%$ & $95.49 \%$ & $87.65 \%$ & $79.14 \%$ & $74.38 \%$ \\
THMI & $97.06 \%$ & $95.25 \%$ & $88.24 \%$ & $78.16 \%$ & $75.01 \%$ \\
KHMI & $97.35 \%$ & $95.47 \%$ & $87.14 \%$ & $80.56 \%$ & $74.64 \%$ \\
CTMI & $98.15 \%$ & $96.24 \%$ & $89.58 \%$ & $81.25 \%$ & $76.98 \%$ \\
\hline
\end{tabular}

\section{Conclusion}

In this paper, we have proposed a new set of Charlier-Tchebichef discrete orthogonal invariant moments. This set of invariant moments is derived algebraically from geometric invariant moments. The invariability and the accuracy of recognition of the proposed CTMI in the classification of the object are carried out and are better than that of TKMI, THMI and KHMI. These moments have desirable image representation capability and can be useful in the field of image analysis.

\section{References}

1. Hu, M.K.: Visual pattern recognition by moment invariants. IRE Trans. Inform. Theory IT-8, 179-187 (1962)

2. Teague, M.R.: Image analysis via the general theory of moments. J. Opt. Soc. Amer. 70, 920-930 (1980)

3. Hosny, K.M.: Image representation using accurate orthogonal Gegenbauer moments. Pattern Recognition Letters 32(6), 795-804 (2011)

4. Zhang, H., Shu, H.Z., Haigron, P., Li, B.S., Luo, L.M.: Construction of a complete set of orthogonal Fourier-Mellin moment invariants for pattern recognition applications. Image and Vision Computing 28(1), 38-44 (2010)

5. Khotanzad, Y.H.H.: Invariant image recognition by Zernike moments. IEEE Transactions on Pattern Analysis and Machine Intelligence 12, 489-497 (1990)

6. Teh, C.H., Chin, R.T.: On image analysis by the method of moments. IEEE Trans. Pattern Anal. Mach. Intell. 10(4), 496-513 (1988)

7. Liao, S.X., Pawlak, M.: On image analysis by moments. IEEE Trans. Pattern Anal. Mach. Intell. 18(3), 254-266 (1996) 
8. Mukundan, R., Ong, S.H., Lee, P.A.: Image analysis by Tchebichef moments. IEEE Trans. Image Process 10(9), 1357-1364 (2001)

9. Yap, P.T., Paramesran, R., Ong, S.H.: Image analysis by Krawtchouk moments. IEEE Transactions on Image Processing 12(11), 1367-1377 (2003)

10. Sayyouri, M., Hmimd, A., Qjidaa, H.: A fast computation of Charlier moments for binary and gray-scale images. In: Information Science and Technology Colloquium (CIST), Fez, Morocco, October 22-24, pp. 101-105 (2012)

11. Yap, P.T., Raveendran, P., Ong, S.H.: Image analysis using Hahn moments. IEEE Trans. Pattern Anal. Mach. Intell. 29(11), 2057-2062 (2007)

12. Sayyouri, M., Hmimid, A., Qjidaa, H.: Improving the performance of image classification by Hahn moment invariants. J. Opt. Soc. Am. A 30, 2381-2394 (2013)

13. Sayyouri, M., Hmimd, A., Qjidaa, H.: A Fast Computation of Hahn Moments for Binary and Gray-Scale Images. In: IEEE International Conference on Complex Systems ICCS 2012, Agadir, Morocco, November 5-6, pp. 1-6 (2012)

14. Mukundan, R.: Some computational aspects of discrete orthonormal moments. IEEE Transactions on Image Processing 13(8), 1055-1059 (2004)

15. Zhu, H., Liu, M., Shu, H., Zhang, H., Luo, L.: General form for obtaining discrete orthogonal moments. IET Image Process. 4(5), 335-352 (2010)

16. Zhu, H.: Image representation using separable two-dimensional continuous and discrete orthogonal moments. Pattern Recognition 45(4), 1540-1558 (2012)

17. Tsougenis, E.D., Papakostas, G.A., Koulouriotis, D.E.: Introducing the Separable Moments for Image Watermarking in a Totally Moment-Oriented Framework. In: Proceedings of the 18th International Conference on Digital Signal Processing (DSP 2013), Santorini, Greece, July 1-3, pp. 1-6 (2013)

18. Tsougenis, E.D., Papakostas, G.A., Koulouriotis, D.E.: Image Watermarking via Separable Moments. Multimedia Tools and Applications (in press)

19. Nikiforov, A.F., Suslov, S.K., Uvarov, B.: Classical orthogonal polynomials of a discrete variable. Springer, New York (1991)

20. Koekoek, R., Lesky, P.A., Swarttouw, R.F.: Hypergeometric orthogonal polynomials and their q-analogues. Springer Monographs in Mathematics. Library of Congress Control Number: 2010923797 (2010)

21. http://www. cs.columbia.edu/CAVE/software/softlib/coil-20.php

22. http://www. dabi.temple. edu/ shape/MPEG7/dataset.html

23. Mukundan, R., Ramakrishnan, K.R.: Moment Functions in Image Analysis. World Scientific Publisher, Singapore (1998) 